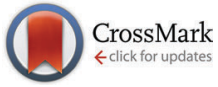

Cite this: Chem. Commun., 2015, 51, 13397

Received 2nd June 2015, Accepted 15th July 2015

DOI: $10.1039 / c 5 c c 04523 a$

www.rsc.org/chemcomm

\section{Engineering polypeptide folding through trans double bonds: transformation of miniature $\beta$-meanders to hybrid helices $\uparrow$}

\author{
Mothukuri Ganesh Kumar, ${ }^{a}$ Sushil N. Benke, ${ }^{a}$ K. Muruga Poopathi Raja*b and \\ Hosahudya N. Gopi*a
}

\begin{abstract}
Utilization of conjugated double bonds to engineer the novel folded miniature $\beta$-meander type structures, single step transformation of miniature $\beta$-meanders into $\alpha \alpha \gamma^{4}$-hybrid peptide 10/12-helices using catalytic hydrogenation, their solution and single crystal conformations are reported.
\end{abstract}

Engineering the folding of polypeptides into defined structures has a great significance from the perspective of medicinal chemistry, biology and materials science. Enormous efforts have been made in this regard to design novel folded architectures to mimic protein secondary structures using a variety of organic templates and synthetic backbone homologated $\beta$ and $\gamma$-amino acids. ${ }^{1,2}$ The homo and heterooligomers of $\beta$ - and $\gamma$-amino acids have displayed a variety of helical patterns and based on the intramolecular H-bonding pseudocycles they are denoted as $\mathrm{C}_{n}$-helices, where $n$ is $14,13,12$ etc. ${ }^{3}$ In addition to the helices, these unnatural oligomers have also been used to mimic $\beta$-sheets ${ }^{4}$ and turns. ${ }^{5}$ However, design of highly organized protein supersecondary structures such as helix-turn-helix, $\beta$-meanders, Greek key motifs etc. from unnatural oligomers has been scarcely investigated.

We have been interested in the utilization of naturally occurring functionalized $\gamma$-amino acids such as statines ${ }^{6}$ and $\alpha, \beta$-unsaturated $\gamma$-amino acids ${ }^{7}$ in the design of foldamers. We recently showed stable $\beta$-hairpin ${ }^{8}$ and unusual planar structures in hybrid peptides containing $\alpha$ - and $E$-vinylogous amino acids. ${ }^{9}$ We foresee that the exceptional conformational properties of $E$-vinylogous amino acids can be explored to design highly organized protein supersecondary structure mimetics beyond the conventional secondary structures (helix, $\beta$-sheets and turns). We postulated that the $\beta$-sheet promoting ${ }^{8,10}$ and turn ${ }^{11}$ inducing properties of $E$-vinylogous

\footnotetext{
${ }^{a}$ Department of Chemistry, Indian Institute of Science Education and Research, Dr. Homi Bhabha Road, Pune-411 008, India. E-mail: hn.gopi@iiserpune.ac.in ${ }^{b}$ Department of Physical Chemistry, School of Chemistry, Madurai Kamaraj University, Madurai-625 021, India. E-mail: murugapoopathiraja@gmail.com $\dagger$ Electronic supplementary information (ESI) available: Experimental details, ${ }^{1}$ H NMR; 2D-NMR, and crystallographic information. CCDC 1063358 (P1), 1060827 (P3) and 1060826 (P4). See DOI: 10.1039/c5cc04523a
}

amino acids can be readily exploited in the design of miniature $\beta$-meander mimetics. The $\beta$-meander is a common protein supersecondary structural motif consisting of two or more anti-parallel $\beta$-strands and each adjacent strand is connected by $\beta$-turns. ${ }^{12}$ The $\beta$-meander structural motif is frequently observed in many proteins including proteases and enzymes. ${ }^{13}$ Interestingly, $\beta$-barrel and $\beta$-propeller structural motifs are constituted from $\beta$-meander topology. ${ }^{14}$ We envisage that by introducing the conformational constraints through $E$-vinylogous amino acids after every two $\alpha$-residues in a hybrid peptide sequence it is possible to construct sheet and reverse turns similar to that of $\beta$-meanders. Herein, we report the designed novel miniature $\beta$-meanders from $\alpha \alpha E$-vinylogous hybrid hexapeptides, single step transformation of miniature $\beta$-meanders into 10/12 $\alpha \alpha \gamma$-hybrid helices using catalytic hydrogenation, their solution and single crystal conformations.

To understand the conformational behavior of hybrid peptides composed of $\alpha$ - and $\alpha, \beta$-unsaturated $\gamma$-amino acids, we designed two hexapeptides $\mathbf{P 1}$ and $\mathbf{P 2}$ composed of repeated $\alpha \alpha E$-vinylogous amino acid sequences (Scheme 1). Peptides P1 and P2 consist of $\alpha, \beta$-unsaturated $\gamma$-phenylalanine and $\alpha, \beta$-unsaturated $\gamma$-alanine, respectively. Peptides were synthesized using Boc-chemistry by a solution phase using DCC/HOBt coupling conditions. Pure peptides were subjected to conformational analysis in solution as well as to grow single crystals in the combination of various solvent mixtures.

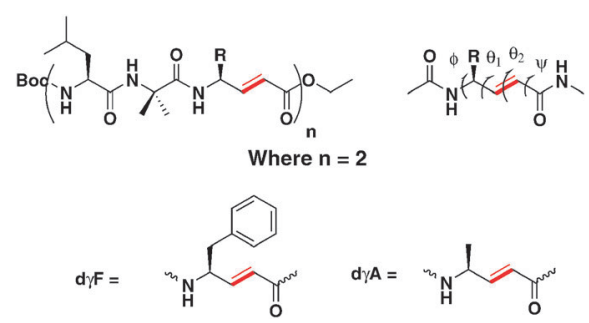

Scheme 1 Sequences of P1 (Boc-Leu-Aib-d $\gamma$ F-Leu-Aib-d $\gamma$ F-OEt) and P2 (Boc-Leu-Aib-d $\gamma$ A-Leu-Aib-d $\gamma$ A-OEt). Local torsional variables of $\alpha, \beta$-unsaturated $\gamma$-residues are also shown. 
Single crystals of $\mathbf{P 1}$ obtained from the slow evaporation of a methanol/toluene solution yielded the structure shown in Fig. 1. Peptide $\mathbf{P 2}$ did not give X-ray quality single crystals. Instructively, $\mathbf{P 1}$ adopted a novel miniature $\beta$-meander type of structural motif with two reverse-turns. The analysis of the crystal structure of $\mathbf{P 1}$ reveals that two $\mathrm{C}=\mathrm{O} \cdots \mathrm{NH}$ intramolecular hydrogen bonds between the urethane carbonyl and the $\mathrm{NH}$ of Leu4 $(1 \leftarrow 4)$ and between the carbonyl of $d \gamma \mathrm{F} 3$ and $\mathrm{NH}$ of $\mathrm{d} \gamma \mathrm{F} 6$ $(1 \leftarrow 4)$ stabilize the overall folding of the molecule. The torsional angles of $\alpha, \beta$-unsaturated $\gamma$-amino acids were measured by introducing two new additional variables $\theta_{1}$ and $\theta_{2}$ along with $\phi$ and $\psi$ (Scheme 1). The $\mathrm{H}$-bond parameters and torsional angles of $\mathbf{P 1}$ are tabulated in the ESI. $\dagger$ Inspection of the crystal structure reveals that Aib2 and $\mathrm{d} \gamma \mathrm{F} 3$ adopted a reverse turn type of conformation stabilized by a 15 -membered H-bond. Furthermore, analysis of the central segment, Leu4-Aib5, reveals that it adopted a type II $\beta$-turn conformation by displaying torsional values $\phi_{4}=-48^{\circ}, \psi_{4}=128^{\circ}, \phi_{5}=68^{\circ}$ and $\psi_{5}=11^{\circ}$ [for a typical type II $\beta$-turn $\phi(i)=-60^{\circ}, \psi(i)=120, \phi(i+1)=80^{\circ}$ and $\left.\psi(i+1)=0^{\circ}\right]$. The type II $\beta$-turn is stabilized by the $1 \leftarrow 4$ hydrogen bond between the carbonyl of $\mathrm{d} \gamma \mathrm{F} 3$ and $\mathrm{NH}$ of $\mathrm{d} \gamma \mathrm{F} 6$.

In order to understand the conformations of P1 and P2 in solutions, we recorded 2D NMR (TOCSY, ROESY and COSY) in $\mathrm{CDCl}_{3}$. The ${ }^{1} \mathrm{H}$ NMR reveals that all amide NHs are in the range of $\delta 6.5-7.3 \mathrm{ppm}$. The amino acid residues and their positions in the peptide sequences were identified using TOCSY and ROESY spectra, respectively. The strong sequential NH $\leftrightarrow \mathrm{NH}$ connectivity between Aib2 $\leftrightarrow \mathrm{d} \gamma \mathrm{F} 3$ and Aib5 $\leftrightarrow \mathrm{d} \gamma \mathrm{F} 6$ residues observed in P1 suggests the reverse-turn conformations of dipeptide segments in the hexapeptide. In addition, a strong NOE between the $\mathrm{d} \gamma \mathrm{F} 3 \mathrm{C}_{\alpha} \mathrm{H}$ and Leu4 $\mathrm{NH}$ indicates the extended conformation of the conjugated amide. The solution conformation analysis of $\mathbf{P 2}$ also reveals a similar type of NOE pattern. Adding to the characteristic sequential $\mathrm{NH} \leftrightarrow \mathrm{NH}$ NOEs between Leu1 $\leftrightarrow$ Aib2, Aib2 $\leftrightarrow \mathrm{d} \gamma$ A3 and Aib5 $\leftrightarrow \mathrm{d} \gamma$ A6, the peptide also showed the NOEs between Aib2 side chains with both Leu1 $\mathrm{NH}$ as well as $\mathrm{NH}$ of $\mathrm{d} \gamma \mathrm{A} 3$. The long range strong NOE is observed between $\mathrm{d} \gamma \mathrm{A} 3 \beta \mathrm{H}$ and the $\mathrm{d} \gamma \mathrm{A} 6$ side-chain which further supports a well folded conformation of $\mathbf{P 2}$ in solution. Using unambiguous NOEs and variable temperature data, solution structure models of $\mathbf{P 1}$ and $\mathbf{P 2}$ are generated and shown in Fig. 1. Instructively, both peptides adopt distinctively different and a spectacular 3-dimentional folded miniature $\beta$-meander type of structures in solution as compared to the 2-dimentional flat crystal structure. We understand that the crystal packing forces possibly directed the 3-dimentional architecture of P1 in single crystals. Overall, the crystal structure and the NMR models suggested that both P1 and P2 adopted novel and distinct miniature $\beta$-meander type of structures.

To appreciate the conformations of their saturated analogues, we subjected both P1 and P2 to catalytic hydrogenation using $20 \% \mathrm{Pd} / \mathrm{C}$ in ethanol. The complete conversion of unsaturated hybrid peptides P1 and P2 into their saturated analogues P3 [BocLeu-Aib- $\gamma^{4}$ Phe-Leu-Aib- $\gamma^{4}$ Phe-OEt] and P4 [Boc-Leu-Aib- $\gamma^{4}$ Ala-LeuAib- $\gamma^{4}$ Ala-OEt], respectively, was achieved within six hours and isolated with $92-95 \%$ yield (Scheme 2). This transformation also

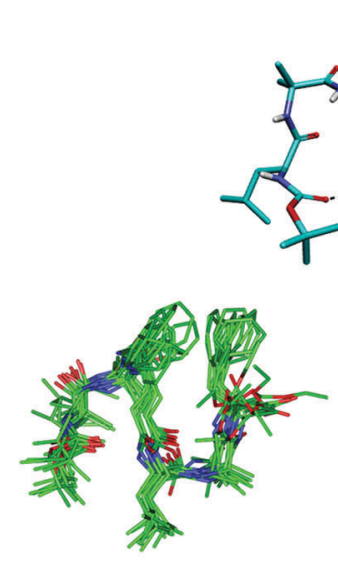

B)

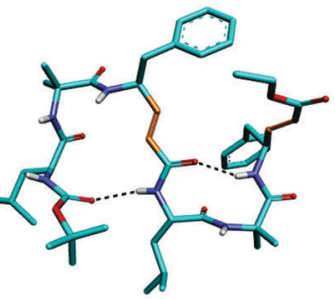

A)

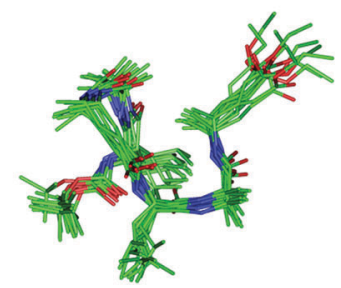

C)
Fig. 1 (A) X-ray structure of peptide P1. (B) NMR model of peptide P1. (C) NMR model of peptide $\mathbf{P 2}$.

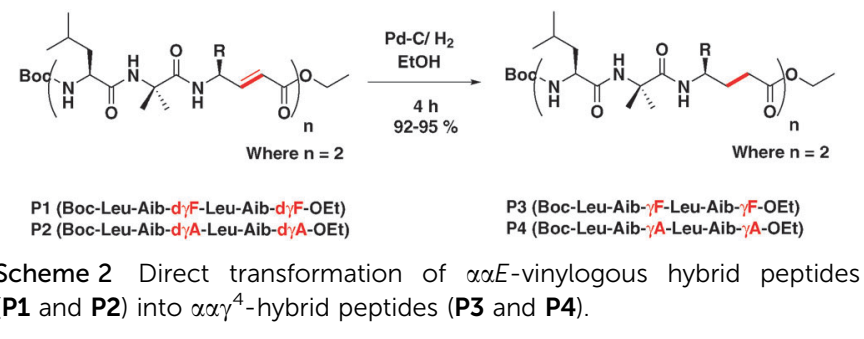

provides an alternative opportunity to synthesize hybrid $\gamma$-peptides without starting from saturated $\gamma$-amino acids. To understand their conformations, both peptides were subjected to crystallization in various solvent combinations. Both P3 and P4 gave X-ray quality single crystals in an aqueous methanol solution and their X-ray structures are shown in Fig. 2. Both peptides adopted helical conformations in single crystals. The release of the geometrical constraints of vinylogous double bonds in the miniature $\beta$-meanders leads to the hybrid helices. Analysis of the crystal structure of P3 reveals that the helical structure is stabilized by four intramolecular $\mathrm{H}$-bonds between $i$ and $i+3$ residues similar to the $3_{10}$-helix ${ }^{15}$ and the $\alpha, \gamma$-hybrid peptide 12-helix, ${ }^{16}$ however with 10 - and 12-membered H-bonds. The 10-membered $\mathrm{H}$-bonds were observed at both $\mathrm{N}$ - and $\mathrm{C}$-termini of the helix, while two consecutive 12-membered H-bonds were observed at the centre of the helix. Similar to P3, P4 also adopted a 10/12 helical structure. These helical structures of $\alpha \alpha \gamma$-hybrid peptides are consistent with the hybrid helices of $-(\alpha \gamma \alpha)_{n}-$ sequences reported by Balaram and colleagues. ${ }^{17}$ The stereochemical analysis of $\gamma^{4}$-residues in both P3 and P4 adopted gauche $e^{+}$gauche ${ }^{+}\left(g^{+}, g^{+}, \theta_{1} \approx \theta_{2} \approx 60^{\circ}\right)$ conformation about the $\mathrm{C}_{\beta}-\mathrm{C}_{\gamma}$ and $\mathrm{C}_{\alpha}-\mathrm{C}_{\beta}$ bonds, while the C-terminal $\gamma$-residues displayed gauche ${ }^{+}\left(\mathrm{C}_{\beta}-\mathrm{C}_{\gamma}\right)$, anti $\left(\mathrm{C}_{\alpha}-\mathrm{C}_{\beta}\right)$ conformation due to the lack of a terminal $\mathrm{H}$-bond donor $(\mathrm{NH})$ to participate in the helix. Similar to the $E$-vinylogous amino acids the torsional angles of $\gamma$-amino acids were measured by introducing additional torsional variables $\theta_{1}$ and $\theta_{2}$ along with $\phi$ and $\psi$. The $\mathrm{H}$-bond parameters and torsional angels of $\gamma$-residues are tabulated in 


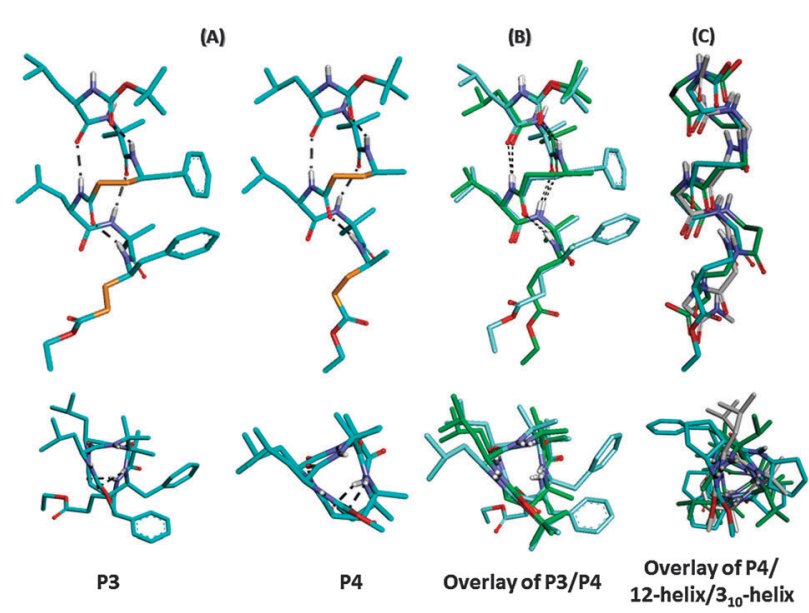

Fig. 2 (A) X-ray structures of peptide P3, P4, (B) superimposition of P3 on $\mathbf{P 4}$ and (C) superimposition of $\mathbf{P 4}$ on $\beta$-peptide 12 -helix and $3_{10}$-helix. Projection of the side-chains is shown in the top-view of the helices.

the ESI. $\dagger$ Interestingly, the top view of the helical peptides in $\alpha \alpha \gamma$-hybrid peptides showed the projection of side-chains at three faces of the helical cylinder similar to the $\beta$-peptide 12-helices and $3{ }_{10}$-helix. ${ }^{18}$ In contrast, $\alpha \gamma$-hybrid peptide 12 -helices showed the projection of the side-chains at four corners of the helical cylinder. ${ }^{14 c}$ The backbone and side-chain correlation of 10/12 hybrid helices with 310 -helix and $\beta$-peptide 12 -helix is shown in Fig. 2C. Analysis suggests that the $\alpha \alpha \gamma$-hybrid peptides can mimic $\beta$-peptide 12 -helices as well as $3_{10}$-helices.

In conclusion, we presented the utilization of geometrically constrained trans double bonds to engineer folding in peptides. The solution and crystal conformations suggested that the designed unsaturated hybrid peptides P1 and P2 adopted a novel miniature $\beta$-meander type of structure. The miniature $\beta$-meander type structure obtained from P1 and P2 offers a glimpse of the potential to generate higher order structures using $\alpha, \beta$-unsaturated $\gamma$-amino acids as guests in host $\alpha$-peptides. Furthermore, these peptides also provide a prospect to transform them into hybrid helices through catalytic hydrogenation. The structural analogy of $\alpha \alpha \gamma$-hybrid 10/12 helices suggests that they can be used to mimic $\beta$-peptide 12 -helices as well as $3_{10}$ helices. As peptide foldamers have been emerging as promising candidates in medicinal chemistry and chemical biology, novel miniature $\beta$-meanders and $\alpha \alpha \gamma$-hybrid peptide helices reported here can be further explored to design functional hybrid peptide foldamers.

K.M.P.R. acknowledges DST-SERB, Govt. of India, for the financial support. M.G.K. and S.N.B. are thankful to CSIR and UGC, respectively, for senior research fellowship.

\section{Notes and references}

1 (a) G. Guichard and I. Huc, Chem. Commun., 2011, 47, 5933; (b) C. G. Cummings and A. D. Hamilton, Curr. Opin. Chem. Biol., 2010, 14, 341.

2 (a) D. Seebach, A. K. Beck and D. J. Bierbaum, Chem. Biodiversity, 2004, 1, 1111; (b) R. P. Cheng, S. H. Gellman and W. F. DeGrado, Chem. Rev., 2001, 101, 3219; (c) T. A. Martinek and F. Fulop, Chem. Soc. Rev., 2012, 41, 687; (d) P. G. Vasudev, S. Chatterjee, N. Shamala and P. Balaram, Chem. Rev., 2011, 111, 657; (e) L. K. A. Pilsl and O. Reiser, Amino Acids, 2011, 41, 709; $(f)$ F. Bouillere, F. ThetiotLaurent, C. Kouklovsky and V. Alezra, Amino Acids, 2011, 41, 687.

3 (a) W. S. Horne and S. H. Gellmann, Acc. Chem. Res., 2008, 41, 1399; (b) G. V. M. Sharma, P. Nagendar, P. Jayaprakash, P. R. Krishna, K. V. S. Ramakrishna and A. C. Kunwar, Angew. Chem., Int. Ed., 2005, 44, 5878; $(c)$ N. Pendem, Y. R. Nelli, C. Douat, L. Fischer, M. Laguerre, E. Ennifar, B. Kauffmann and G. Guichard, Angew. Chem., Int. Ed., 2013, 52, 4147; (d) S. Chatterjee, R. S. Roy and P. Balaram, J. R. Soc., Interface, 2007, 4, 587.

4 (a) I. L. Karle, H. N. Gopi and P. Balaram, Proc. Natl. Acad. Sci. U. S. A., 2002, 99, 5160; (b) G. A. Lengyel, R. C. Frank and W. S. Horne, J. Am. Chem. Soc., 2011, 133, 4246; (c) L. Sebaoun, V. Maurizot, T. Granier, B. Kauffmann and I. Huc, J. Am. Chem. Soc., 2014, 136, 2168.

5 (a) D. Seebach, S. Abele, K. Gademann and B. Jaun, Angew. Chem., Int. Ed., 1999, 38, 1595; (b) S. Chatterjee, P. G. Vasudev, S. Raghothama, C. Ramakrishnan, N. Shamala and P. Balaram, J. Am. Chem. Soc., 2009, 131, 5956.

6 (a) B. M. Dunn, Chem. Rev., 2002, 102, 4431; (b) D. Leung, G. Abbenante and D. P. Fairlie, J. Med. Chem., 2000, 43, 305; (c) A. Bandyopadhyay, A. Malik, M. Ganesh Kumar and H. N. Gopi, Org. Lett., 2014, 16, 294.

7 (a) R. G. Linington, B. R. Clark, E. E. Trimble, A. Almanza, L.-D. Urena, D. E. Kyle and W. H. Gerwick, J. Nat. Prod., 2009, 72, 14; (b) M. Hagihara and S. L. Schreiber, J. Am. Chem. Soc., 1992, 114, 6570.

8 A. Bandyopadhyay, S. M. Mali, P. Lunawat, K. M. P. Raja and H. N. Gopi, Org. Lett., 2011, 13, 4482.

9 A. Bandyopadhyay and H. N. Gopi, Org. Lett., 2012, 14, 2770.

10 M. Hagihara, N. J. Anthony, T. J. Stout, J. Clardy and S. L. Schreiber, J. Am. Chem. Soc., 1992, 114, 6568.

11 (a) T. K. Chakraborty, A. Ghosh, S. K. Kumar and A. C. Kunwar, J. Org. Chem., 2003, 68, 6459; (b) C. Grison, P. Coutrot, S. Geneve, C. Didierjean and M. Marraud, J. Org. Chem., 2005, 70, 10753.

12 T. E. Creighton, Proteins: structure and molecular properties, 2nd edn, W. H. Freeman and Co., New York, 1993, p. 227.

13 D. N. Woolfson, G. J. Bartlett, A. J. Burton, J. W. Heal, A. Niitsu, A. R. Thomson and C. W. Wood, Curr. Opin. Struct. Biol., 2015, 33, 16.

14 (a) S. Balaji, Curr. Opin. Struct. Biol., 2015, 32, 156; (b) W. C. Wimley, Curr. Opin. Struct. Biol., 2003, 13, 404; (c) G. E. Schulz, Curr. Opin. Struct. Biol., 2000, 10, 443; (d) C. Zhang and S.-H. Kim, J. Mol. Biol., 2000, 299, 1075.

15 (a) I. L. Karle and P. Balaram, Biochemistry, 1990, 29, 6747; (b) C. Toniolo, M. Crisma, F. Formaggio and C. Peggion, Biopolymers, 2001, 60, 396.

16 (a) S. Chatterjee, P. G. Vasudev, S. Raghothama, C. Ramakrishnan, N. Shamala and P. Balaram, J. Am. Chem. Soc., 2009, 131, 5956; (b) L. Guo, Y. Chi, A. M. Almeida, I. A. Guzei, B. K. Parker and S. H. Gellman, J. Am. Chem. Soc., 2009, 131, 16018; (c) A. Bandyopadhyay, S. V. Jadhav and H. N. Gopi, Chem. Commun., 2012, 48, 7170.

17 K. Basuroy, B. Dinesh, N. Shamala and P. Balaram, Angew. Chem., Int. Ed. Engl., 2013, 52, 3136.

18 (a) C. Crisma, M. Saviano, A. Moretto, Q. B. Broxterman, B. Kaptein and C. Toniolo, J. Am. Chem. Soc., 2007, 129, 15471; (b) S. H. Choi, I. A. Guzei, L. C. Spencer and S. H. Gellman, J. Am. Chem. Soc., 2010, 132, 13879. 